\title{
Essential modifications
}

\author{
Ubiquitin and ubiquitin-like proteins have central roles in regulating cellular processes and homeostasis. \\ This Focus examines our understanding of the ubiquitination reaction and the mechanisms by which ubiquitin \\ and related modifications affect protein and cellular functions.
}

U biquitination, the post-translational modification by which ubiquitin is attached to a protein substrate, is an essential regulatory reaction in all eukaryotes. Proteins can be modified at substrate lysine residues with a single ubiquitin or with a polyubiquitin chain. Ubiquitination can mark a protein for proteasomal degradation, alter its cellular localization, change its activity level, or prevent or promote its interaction with other proteins. In this issue, we present a special Focus on Ubiquitin (http://www.nature.com/nsmb/focus/ ubiquitin) that discusses recent insights regarding the mechanisms of ubiquitination and their regulation and the various roles ubiquitin and ubiquitin-like proteins play in shaping cellular processes.

Ubiquitination is catalyzed by a three-enzyme cascade that results in the formation of an isopeptide linkage between the $\mathrm{C}$ terminus of ubiquitin and the $\varepsilon$-amino group of the target lysine. The cascade is initiated by the E1 enzyme, which activates the ubiquitin $\mathrm{C}$ terminus through a thioester bond that is transferred to the active site cysteine of the $\mathrm{E} 2$ conjugating enzyme. The $\mathrm{E} 3$ ligase binds to the E2 ubiquitin thioester intermediate and the protein substrate, catalyzing transfer of ubiquitin to the target lysine (or, in some cases, the protein $\mathrm{N}$ terminus).

In humans, there are several E1s and tens of E2s but several hundred E3s, reflecting the vast array of proteins

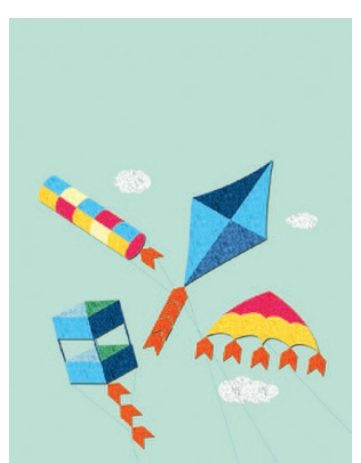

opposing, cellular outcomes. Focusing on the DNA damage tolerance and repair pathways, Ulrich (p 317) reviews the mechanisms by which ubiquitination and SUMOylation can be specifically targeted to DNAor chromatin-bound pools of substrate proteins and the ways that these modifications can then affect the DNA- or chromatin-binding properties of different proteins.

The endoplasmic reticulum-associated degradation (ERAD) pathway is a quality-control system that maintains protein homeostasis in the ER. This subcellular compartment does not contain components of the ubiquitin-proteasome system (UPS), and hence unwanted or misfolded proteins in the ER are retrotranslocated to the cytosol for proteasomal degradation. The retrotranslocation machinery that resides in the ER membrane has integrated components of the UPS, and Christianson and Ye ( $\mathrm{p} 325$ ) consider the role of the ubiquitin system in ERAD, highlighting recent advances in understanding how these two pathways intertwine to facilitate retrotranslocation.

Whereas the UPS degrades individual proteins modified with polyubiquitin chains, autophagy degrades many proteins and organelles en masse. A pair of ubiquitin-like proteins, Atg8 and Atg12, regulates autophagy-mediated degradation in a manner completely distinct from that of ubiquitin in the UPS, as Klionsky and Schulman discuss and biochemical processes with which ubiquitin is associated. The E3 ligases can be divided into three classes-RING, HECT and RBR-that utilize distinct conserved domains and mechanisms to transfer ubiquitin from E2 to substrate. In their Review ( $\mathrm{p} 301$ ), Berndsen and Wolberger discuss the recent explosion in mechanistic and structural understanding of how the three $\mathrm{E} 3$ families enhance substrate ubiquitination and of how E3 ligases are regulated.

Target selection is a critical part of ubiquitin and ubiquitin-like modification cascades. Mattiroli and Sixma ( $\mathrm{p} 308$ ) focus on the subject of lysine specificity in these pathways. It is clear that, for some pathways, the E2 enzyme determines specificity, as is the case for SUMOylation and ubiquitin-chain formation, whereas E3 seems to contribute to specificity in the majority of other pathways. In addition, target selectivity may be dictated by the consequent signal the modification generates: nonselective lysine modifications seem to be sufficient for proteasomal degradation, but a higher degree of specificity is required to initiate a specific signaling cascade.

Ubiquitin and SUMO play significant roles in regulating DNAassociated transactions, including transcription, replication and repair, with each modification signaling for very specific, and sometimes
( $\mathrm{p} 336$ ). Their Review outlines recent progress in understanding how these modifications promote autophagy-associated protein-protein interactions and what roles these ubiquitin-like proteins play in physiology and disease.

The UPS and autophagy are the two major proteolytic pathways used to ensure efficient protein turnover and they are essential for proteostasis. In their Commentary, Kevei and Hoppe (p 290) discuss the impact of age- and stress-induced signals on these ubiquitin-dependent proteolytic pathways. Recent insights into the role of the UPS in plant growth and development are explored in a second Commentary from Shabek and Zheng ( $p$ 293). Finally, Corn and Vucic (p 297) focus on the importance of the UPS in coordinating the inflammatory response, which requires effective and precise interplay between multiple key signaling pathways.

Given the defining ubiquity of ubiquitin and related modifications and their essentiality to cellular function, it is clear that researchers have as yet revealed only the tip of the iceberg in terms of understanding how these modifications act to regulate the myriad number of processes within the cell. We are eager to see more details emerge and develop within the pages of NSMB. Lastly, we would like to thank our sponsor, Takeda, whose generous support allows Focus content to be freely available for the next 6 months. 